\title{
Neurologic Findings in Infants With Deformational Plagiocephaly
}

\author{
Elizabeth A. Fowler, Devra B. Becker, Thomas K. Pilgram, Michael Noetzel, Jay Epstein and Alex \\ A. Kane \\ J Child Neurol 2008; 23; 742 originally published online Mar 14, 2008; \\ DOI: $10.1177 / 0883073808314362$
}

This study evaluates the neurologic profiles of infants with deformational plagiocephaly. Forty-nine infants with deformational plagiocephaly between the ages of 4 and 13 months (mean age, 8.1 months) are evaluated, along with 50 age-matched control subjects (mean age, 8.1 months). A modified version of the Hammersmith infant neurologic assessment was performed on each infant. A caregiver completed a questionnaire regarding the infant's prematurity, development, and health to date. Results are analyzed using $t$ test. There is a statistically significant difference in overall neurologic assessment scores of infants with deformational plagiocephaly vs their healthy peers $(P=.002)$. This difference is predominately in tone, whereby infants with deformational plagiocephaly have significantly more abnormal tone than nonplagiocephalic infants $(P=.003)$. This abnormality is not one of decreased tone but one of variable tone, deflecting abnormally high and low tone. Infants with deformational plagiocephaly are more likely to have altered tone but not exclusively decreased tone.

Keywords: deformational plagiocephaly; neurodevelopment; plagiocephaly

n 1992, the American Academy of Pediatrics introduced the "Back to Sleep" campaign, recommending that infants should be placed supine to reduce the risk of sudden infant death syndrome. Along with a decrease in deaths due to sudden infant death syndrome after the campaign's introduction, there was a subsequent corresponding increase in the number of infants presenting with deformational plagiocephaly. ${ }^{2,3}$ The increase in cases during the past decade has been well documented ${ }^{2,4,5}$ and has led to greater investigation into the pathogenesis and associated abnormalities of deformational plagiocephaly.

Historically, deformational plagiocephaly has been considered an abnormality associated with benign craniofacial asymmetry, of primary significance in that it alters appearance. In recent years, however, subtle neurologic abnormalities have been reported to accompany deformational plagiocephaly in infancy and later in life. School-aged

children with deformational plagiocephaly have been shown to be more likely to need special help during primary school in areas such as special education, physical therapy, occupational therapy, and speech therapy. ${ }^{6}$ Kordestani et al demonstrated that infants with deformational plagiocephaly showed significant delays in mental and psychomotor development and that none of 110 infants studied showed accelerated development. It has also been shown that mothers of infants diagnosed as having deformational plagiocephaly were more likely to report a perceived 
developmental delay and lower activity level of their infant than parents of nonplagiocephalic infants. Davis et al ${ }^{8}$ demonstrated that infants with deformational plagiocephaly reached motor milestones later compared with their healthy peers. This has led to much speculation surrounding the neurologic implications of deformational plagiocephaly and the role of motor tone in the development of deformational plagiocephaly. Abnormal motor tone, specifically hypotonia, has been lumped into the list of possible causes of deformational plagiocephaly, but no research to date has explored this relationship. ${ }^{10}$ The present study evaluated the comprehensive neurologic profiles of infants with deformational plagiocephaly compared with their healthy peers, focusing on development, tone, and posture. We do not endeavor to establish causality between tone and deformational plagiocephaly, nor do we investigate treatment modalities or outcomes; rather, we characterize the neurologic profile of children with deformational plagiocephaly at the time of diagnosis.

\section{Methods}

After approval from the Washington University Human Studies Committee, 49 patients with radiographically confirmed deformational plagiocephaly, aged 4 to 13 months, were recruited during a 2-month period. Concurrently, 50 nonplagiocephalic infants (as determined by visual inspection and review of medical history) of similar age were recruited during well-child visits at a nearby pediatrician's office. When available, each child's gestational age, as recorded in their medical record, was noted.

\section{Ages and Stages Questionnaires}

The primary parent available completed the age-appropriate Ages and Stages Questionnaires. The Ages and Stages Questionnaires consist of a series of age-appropriate surveys regarding psychomotor development to be completed by parents and caregivers of children aged 4 to 48 months. The series of surveys has been shown to demonstrate excellent interobserver reliability and validity of $85 \%$." Questionnaires were available for only 4-, 6-, 8-, 10-, and 12-month intervals; infants falling between 2 age groups were moved up to the nearest grouping. Each survey contained questions designed to evaluate parental perception of infant communication and gross motor, fine motor, problem solving, and personal-social development. Questionnaires were scored according to Ages and Stages Questionnaires guidelines. A "yes" response received a score of 10 points; "sometimes," 5 points; and "not yet," 0 points. Blank responses were scored by averaging all answered questions in the section. If more than 2 questions in a given section were left unanswered, that section was not scored. Sectional scores were obtained by summing questions in each section. In addition, birth dates, corrected birth dates, and overall health were noted.

\section{Hammersmith Infant Neurologic Assessment}

Behavioral state and nonrevised optimality scoring. Each infant was evaluated by a single examiner using a modified version of the Hammersmith infant neurologic assessment for infants aged 2 to 24 months. ${ }^{12}$ Variables (Table 1) concerning posture, tone, and behavior were assessed individually and were assigned optimality scores at 12 and 18 months of age as determined by the distribution of frequency of findings in a healthy population in the manner described by Haataja et al. ${ }^{1,13}$ Posture and tone variables were scored out of a possible 3 points. A score of 3 corresponded to the finding observed in at least $75 \%$ of the healthy population, a score of 2 to that observed in fewer than $25 \%$ but in at least $10 \%$, and a score of 1 or 0 to that seen in fewer than $10 \%$ of the population, which represents varying degrees of severity, with 0 being the most severe. Behavior variables (social orientation, emotional state, and consciousness) were scored using descriptive scoring rubrics with scales of 1 to 4,1 to 5 ,

Table 1. Variables Assessed in 3 Categories

Posture Head Trunk Arms Legs (supine and sitting) Feet (supine and sitting)

Tone Scarf sign ${ }^{\mathrm{a}}$ Passive shoulder resistance Wrist pronation or supination Adductor angle Popliteal angle Ankle dorsiflexion ${ }^{\mathrm{a}}$ Head lag when pulled to sit Ventral suspension

Behavior Social orientation Emotional state Consciousness

a. Right and left evaluated and scored independently. 
and 1 to 6 , respectively, and were included to assess possible confounding due to variations in behavioral state.

Sectional optimality scores were obtained by summing the individual scores for a given section. Global optimality scores were obtained by summing all variables for a given individual. If an individual variable failed to be scored because of infant noncooperation, it was assigned the average of the remaining variables in that section when calculating the sectional and global optimality scores.

Revised tone scoring. The original Hammersmith infant neurologic assessment optimality scoring system rates infants' tone on a scale ranging from normal (seen in $>75 \%$ of the population) to abnormal (seen in $<10 \%$ ). To more fully examine the character of the infants' tone, the scoring of the tone section in the Hammersmith infant neurologic assessment was prospectively revised to evaluate the infants' tone as increased, normal, or decreased. In the revised tone scoring, negative scores denote decreased tone, 0 denotes normal tone, and positive scores denote increased tone. The revised tone scoring sheet is given in Table 2. Total revised tone scores were obtained by summing the single variable scores for each patient.

\section{Statistical Analysis}

Data were analyzed by calculating and comparing means. Differences in means were tested for statistical significance using $t$ test. Analysis was performed using JMP 6.0 (SAS Institute, Cary, North Carolina).

\section{Results}

The data for 49 infants with deformational plagiocephaly and 50 age-matched control subjects were analyzed

Table 2. Revised Tone Scoring Used for More In-depth Analysis of Infant Tone

\begin{tabular}{|c|c|c|c|c|c|c|}
\hline Varable & -2 Low Tone & -1 & -0.5 & 0 Normal & 1 & 2 High Tone \\
\hline \multicolumn{7}{|l|}{ Resistance } \\
\hline Scarf sign & None & Minimal & - & Moderate & - & Severe \\
\hline Passive shoulder & - & None & - & Overcome & - & Not overcome \\
\hline \multicolumn{7}{|l|}{ Range } \\
\hline Adductor angle & - & $>170^{\circ}$ & $150^{\circ}-170^{\circ}$ & $80^{\circ}-150^{\circ}$ & - & $<80^{\circ}$ \\
\hline Popliteal angle & - & $>170^{\circ}$ & $150^{\circ}-160^{\circ}$ & $110^{\circ}-150^{\circ}$ & $<90^{\circ}$ & $<80^{\circ}$ \\
\hline Ankle dorsiflexion & - & $<20^{\circ}$ & $20^{\circ}-30^{\circ}$ & $30^{\circ}-85^{\circ}$ & $90^{\circ}$ & $>90^{\circ}$ \\
\hline \multicolumn{7}{|l|}{ Head Lag } \\
\hline When pulled to sit & Severe & Moderate & - & None & - & - \\
\hline \multicolumn{7}{|l|}{ Back Curvature } \\
\hline Ventral suspension & Severe & Moderate & - & None & - & - \\
\hline
\end{tabular}

Table 3. Ages of Cases and Controls

\begin{tabular}{llllll} 
Group & Age, mean (SD), mo & $95 \%$ Confidence Interval & $\begin{array}{l}\text { Gestational Age, mean } \\
(\text { SD), wk }\end{array}$ & $95 \%$ Confidence Interval & $\begin{array}{l}\text { Gestational Age, } \\
\text { median, wk }\end{array}$ \\
\hline $\begin{array}{l}\text { Cases } \\
\text { Controls } P \\
\text { value }\end{array}$ & $8.1(0.3) 8.1(0.5) .97$ & $7.4-8.87 .2-9.0-$ & $38.7(0.3) 38.6(0.2) .72$ & $38.2-39.338 .1-39.1-$
\end{tabular}

( $100 \%$ of those who chose to participate). The gestational ages were collected for all case infants and for 48 of 50 control infants. (Two infants had no medical record notation of gestational age.) The mean ages and gestational ages for the 2 groups were almost identical (Table 3 ).

\section{Ages and Stages Questionnaires}


The Ages and Stages Questionnaires data are shown in Figure 1. Infants with deformational plagiocephaly tended to score lower in all categories of the Ages and Stages Questionnaires. However, this trend was only statistically significant for personal-social skills $(P<.001)$.

\section{Hammersmith Infant Neurologic Assessment}

Behavioral state and nonrevised optimality scoring. Behavioral state scoring is shown in Figure 2. There was no statistically significant difference with respect to consciousness, emotional state, and social orientation. The mean overall scores for the Hammersmith infant neurologic assessment are given in Table 4. Although the difference in scores between infants with deformational plagiocephaly and their healthy counterparts was not large, it was statistically significant $(P=.002)$. Figure 3 shows that the difference in nonrevised optimality scores is primarily in tone, whereby infants with deformational plagiocephaly scored lower (indicating more abnormal tone) than their healthy peers $(P=.003)$; posture was not significantly different $(P=.81)$.

Revised tone scoring. The mean values for the revised tone scores are given in Table 5 . Both groups averaged slightly below 0 ; on this scale, 0 indicates normal tone, positive numbers indicate high tone, and negative numbers indicate low tone. Although the means were not significantly different, the cases demonstrate a wider confidence interval than the controls, indicating that their revised tone scores were more variable, stretching higher and lower than those of their healthy counterparts, as shown in Figure 4.

\section{Discussion}

Deformational plagiocephaly, also referred to as positional plagiocephaly or as nonsynostotic plagiocephaly, refers to a constellation of craniofacial asymmetric findings, most notably including unilateral flattening of the occiput, ipsilateral protrusion of the frontal bone, and ipsilateral displacement of the ear anteriorly. ${ }^{14.15}$ Unlike synostotic plagiocephaly, deformational plagiocephaly is often treated with a custom orthotic molding helmet. Deformational plagiocephaly is typically diagnosed when a child is younger than 1 year, when the skull is still rapidly growing and malleable.

Several mechanisms of the skull deformation in deformational plagiocephaly have been proposed. Intrauterine constraint has been shown to cause deformation of the skull, and frontal and occipital plagiocephaly has been reported. ${ }^{8,17-19}$ However, this constraint does not account for the rapid rise in the prevalence of deformational plagiocephaly with occipital flattening, as seen since the

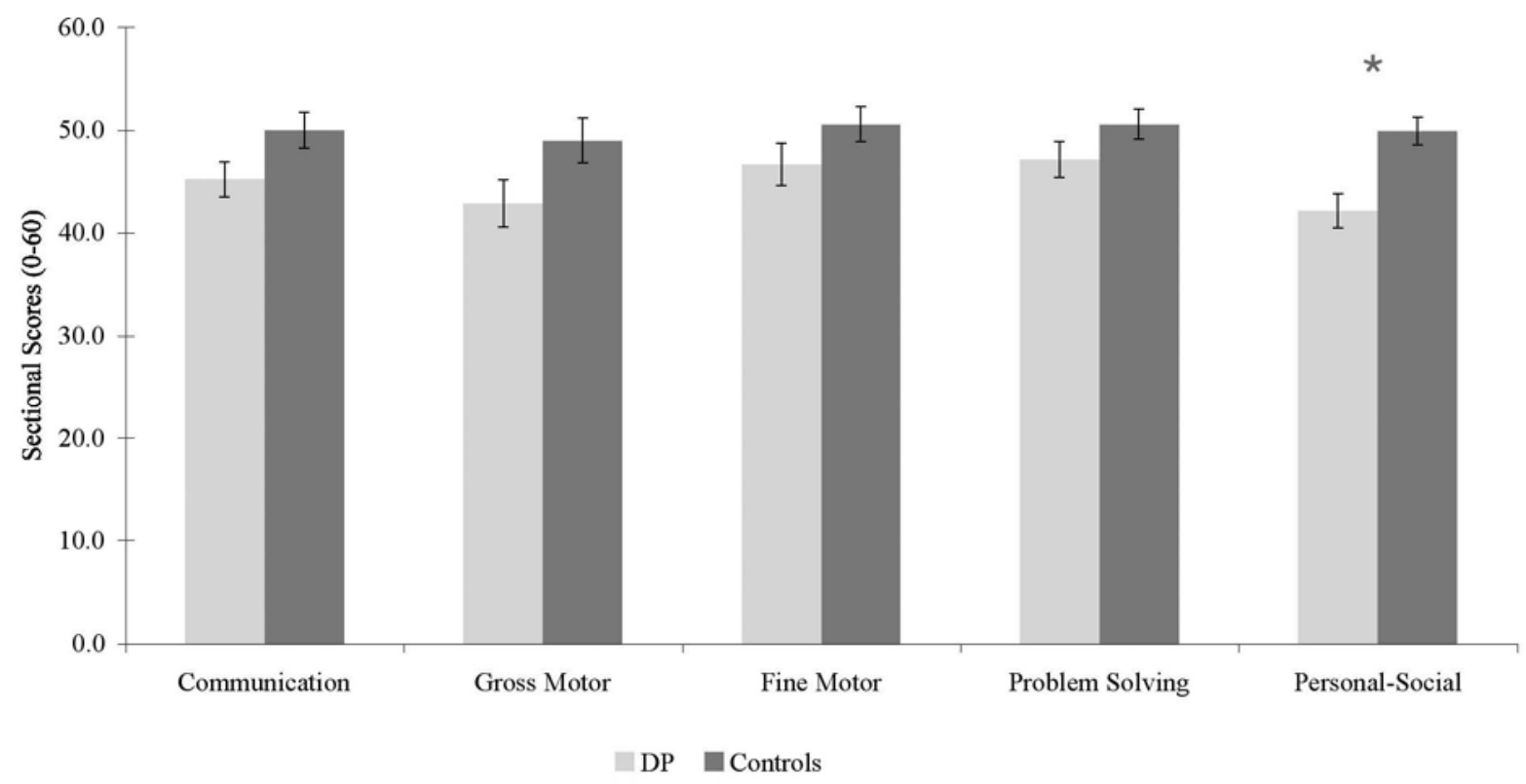


Figure 1. Ages and Stages Questionnaires scores by category for infants with deformational plagiocephaly and for control subjects. ${ }^{*} P<.001$.

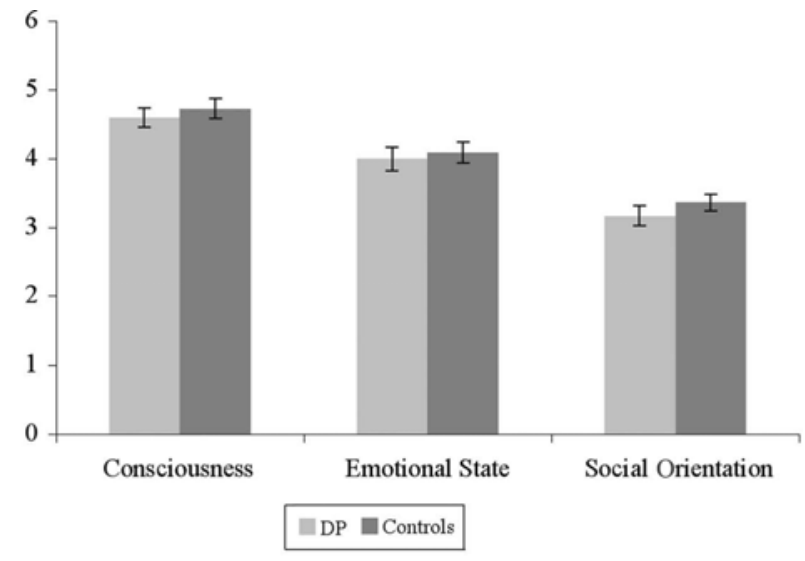

Figure 2. Mean scores for consciousness $(P=.52)$, emotional state $(P=$

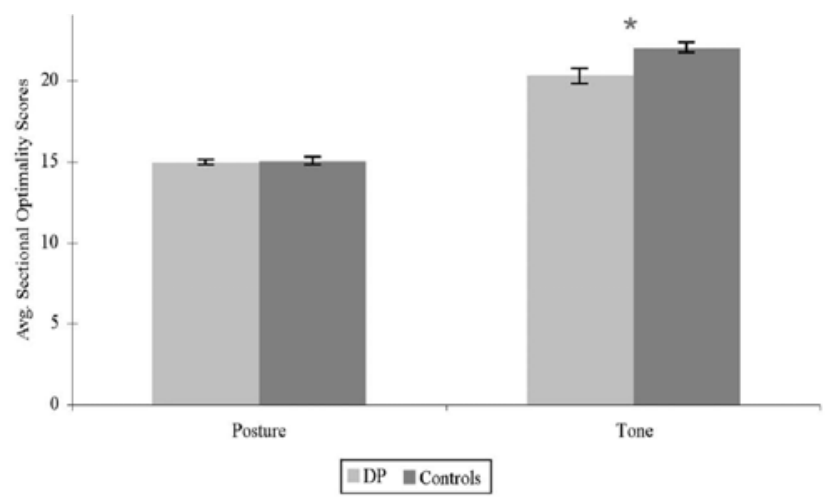

Figure 3. Mean nonrevised optimality scores for posture and for tone among infants with deformational plagiocephaly and among control

$.70)$, and social orientation $(P=.31)$ for infants with deformational plagio

cephaly and for control subjects.

\section{Table 4.}

Hammersmith Infant Neurologic introduction of the "Back to Sleep" campaign. It has been Assessment Scores proposed that the supine sleeping position also plays a causative role in

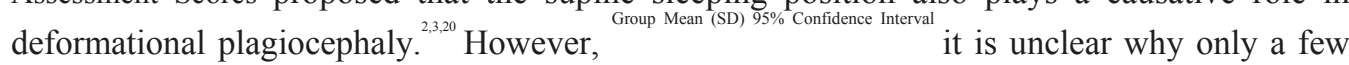
infants develop deformational Cases 40.16 (0.43) 39.30-41.02 plagiocephaly among the many infants who sleep supine. Controls 41.79 (0.30) 41.19-42.39 The Hammersmith infant neurologic assessment, $P$ Value .002

used herein to assess infant posture, tone, and behavior, was first developed as a standard neurologic assessment of preterm neonates and was later revised for use in development of motor function, and state of behavior. older infants aged 2 to 24 months. The original examina-Within the neurologic signs section, there were several subtion included the following 3 sections: neurologic signs, categories designed to individually assess infant cranial

Table 5. Revised Tone Scoring

\begin{tabular}{lcc} 
Group & Mean (SD) & $95 \%$ Confidence Interval \\
\hline Cases & & \\
Controls $P$ & $-0.049(0.433)-0.146$ & -0.919 to $0.822-0.728$ to \\
Value & $(0.290) .85$ & $0.437-$
\end{tabular}

nerve function, posture, movements, tone, and reflexes and reactions. Scoring of the examination is obtained by grading each neurologic finding and is based on the distribution of frequency of findings in a healthy population of children aged 12 to 18 months.

The present study demonstrates significant variation in tone among infants with deformational plagiocephaly compared with their healthy peers. From the Ages and Stages Questionnaires, it seems that infants with deformational plagiocephaly tend to score lower on developmental surveys. However, this trend only reached significance for 
personal-social skills. It is unclear from the present study why this variation in tone did not significantly affect the acquisition of gross motor or fine motor milestones in our study population as recorded by the Ages and Stages Questionnaires. When closely analyzed infants with deformational plagiocephaly obtain motor milestones later than their peers but are still within the normal range, such subtle differences may not be fully evident with the Ages and Stages Questionnaires, and the abnormality in tone could lead to the observed trends that were demonstrated herein.

Infants with deformational plagiocephaly also score lower on the Hammersmith infant neurologic assessment. The lower overall score denoting abnormality predominantly stems from the infants' tone examinations, whereby infants with deformational plagiocephaly demonstrate significantly more abnormal tone than their healthy peers. Finally, as demonstrated by the revised tone scoring, tone is not characterized solely by increased or decreased tone but by more widely variable tone among the infants with deformational plagiocephaly, as indicated by the width of the confidence interval. This variability is an important finding in that hypotonia, but not hypertonia, has been considered a determining factor in the development of deformational plagiocephaly. ${ }^{1021}$ It is unclear from this study why infants with deformational plagiocephaly display more variable tone or if this is a causal factor in the development of deformational plagiocephaly. It is feasible that floppiness associated with hypotonia and constrained posture associated with hypertonia could lead to immobilization of the infant head and to plagiocephaly. Further study is needed before this can be determined.

This study has several limitations. Neither the researchers nor the parents were blinded to diagnosis. Furthermore, the study group consisted of parents who sought treatment for a diagnosis of deformational plagiocephaly. It is unknown what

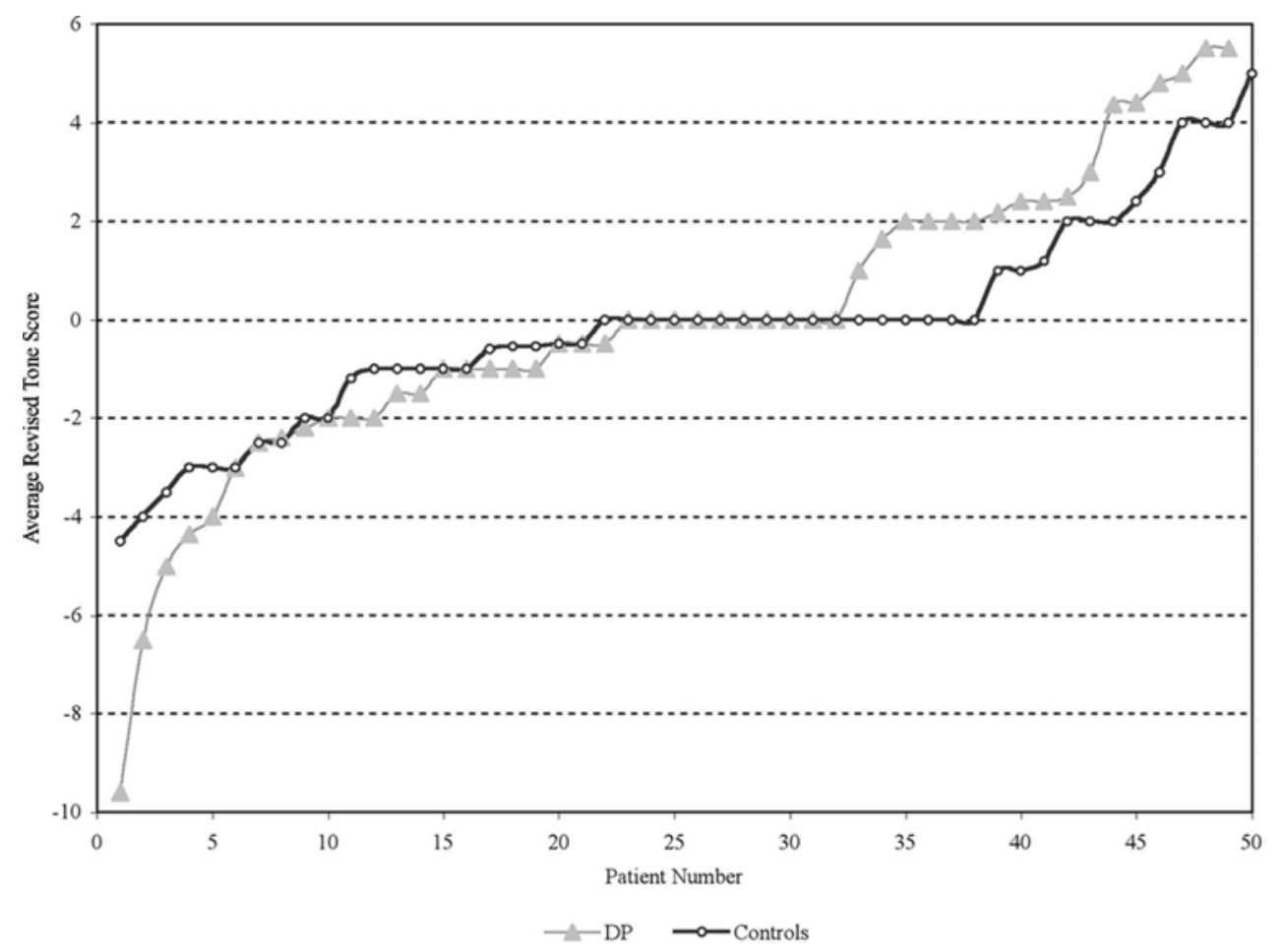

Figure 4. Revised tone scores.

sort of results our study would have shown if we had included a group of children who had been diagnosed as having deformational plagiocephaly but whose parents decided not to pursue treatment. In addition, the methods did not seek to match case and control subjects with regard to socioeconomic or other variables. Also, the study examined infants at several months of age and does not consider tone and movements during the neonatal period, which could potentially affect head shape later in life.

Primarily, these results reveal that tone is significantly altered in infants with deformational plagiocephaly. 
Although this difference is small, it is important in that tone may have a potential confounding effect when interpreting neurodevelopmental differences in infants with plagiocephaly compared with healthy control subjects. For these reasons, tone should continue to be considered an important variable when assessing infants with deformational plagiocephaly. Finally, these results demonstrate a trend toward low scores (indicating abnormal tone) on parental psychomotor evaluation of patients with deformational plagiocephaly.

\section{Acknowledgment}

This study was presented at the 63rd Annual Meeting of the American Cleft Palate and Craniofacial Association; April 58, 2006; Vancouver, British Columbia, Canada.

\section{References}

1 American Academy of Pediatrics AAP Task Force on Infant Positioning and SIDS. Positioning and SIDS [published erratum appears in Pediatrics. 1992;90(2, pt 1):264]. Pediatrics. 1992; 89(6, pt 1):1120-1126.

2 Argenta LC, David LR, Wilson JA, Bell WO. An increase in infant cranial deformity with supine sleeping position. J Craniofac Surg. 1996;7(1):5-11.

3 Kane AA, Mitchell LE, Craven KP, Marsh JL. Observations on a recent increase in plagiocephaly without synostosis. Pediatrics. 1996;97(6, pt 1):877-885.

4 Boere-Boonekamp MM, van der Linden-Kuiper LL. Positional preference: prevalence in infants and follow-up after two years. Pediatrics. 2001;107(2):339-343.

$5 \quad$ Peitsch WK, Keefer CH, LaBrie RA, Mulliken JB. Incidence of cranial asymmetry in healthy newborns. Pediatrics. 2002;110(6):e72.

1 Miller RI, Clarren SK. Long-term developmental outcomes in patients with deformational plagiocephaly. Pediatrics. 2000; 105(2):e26.

2 Kordestani RK, Patel S, Bard DE, Gurwitch R, Panchal J. Neurodevelopmental delays in children with deformational plagiocephaly. Plast Reconstr Surg. 2006;117(1):207-220.

3 Hutchison BL, Thompson JM, Mitchell EA. Determinants of nonsynostotic plagiocephaly: a case-control study. Pediatrics. 2003;112(4):e316.

4 Davis BE, Moon RY, Sachs HC, Ottolini MC. Effects of sleep position on infant motor development. Pediatrics. 1998;102(5): 1135-1140.

5 Balan P, Kushnerenko E, Sahlin P, Huotilainen M, Naatanen R, Hukki J. Auditory ERPs reveal brain dysfunction in infants with plagiocephaly. J Craniofac Surg. 2002;13(4):520-526.

6 Squires J, Bricker D, Potter L. Revision of a parent-completed development screening tool: Ages and Stages Questionnaires. $J$ Pediatr Psychol. 1997;22(3):313-328.

$7 \quad$ Haataja L, Mercuri E, Regev R, et al. Optimality score for the neurologic examination of the infant at 12 and 18 months of age. $J$ Pediatr. 1999;135(2, pt 1):153-161.

$8 \quad$ Haataja L, Cowan F, Mercuri E, Bassi L, Guzzetta A, Dubowitz L. Application of a scorable neurologic examination in healthy term infants aged 3 to 8 months. J Pediatr. 2003; 143(4):546.

9 Clarren SK. Plagiocephaly and torticollis: etiology, natural history, and helmet treatment. J Pediatr. 1981;98(1):92-95.

10 St John D, Mulliken JB, Kaban LB, Padwa BL. Anthropometric analysis of mandibular asymmetry in infants with deformational posterior plagiocephaly. J Oral Maxillofac Surg. 2002;60(8):873-877.

11 Pollack IF, Losken HW, Fasick P. Diagnosis and management of posterior plagiocephaly. Pediatrics. 1997;99(2):180-185.

12 Habal MB, Castelano C, Hemkes N, Scheuerle J, Guilford AM. In search of causative factors of deformational plagiocephaly. $J$ Craniofac Surg. 2004;15(5):835-841.

13 Littlefield TR, Kelly KM, Pomatto JK, Beals SP. Multiple-birth infants at higher risk for development of deformational plagiocephaly. Pediatrics. 1999;103(3):565-569.

14 Littlefield TR, Kelly KM, Pomatto JK, Beals SP. Multiple-birth infants at higher risk for development of deformational plagiocephaly, II: is one twin at greater risk? Pediatrics. 2002;109(1): 19-25.

15 Turk AE, McCarthy JG, Thorne CH, Wisoff JH. The "Back to Sleep Campaign" and deformational plagiocephaly: is there cause for concern? J Craniofac Surg. 1996;7(1):12-18.

16 Panchal J, Amirsheybani H, Gurwitch R, et al. Neurodevelopment in children with single-suture craniosynostosis and plagiocephaly without synostosis. Plast Reconstr Surg. 2001;108(6):1492-1500. 